\title{
A suggested new bacteriophage genus: "Viunalikevirus"
}

\author{
Evelien M. Adriaenssens $\cdot$ Hans-Wolfgang Ackermann $\cdot$ Hany Anany $\cdot$ Bob Blasdel $\cdot$ \\ Ian F. Connerton • David Goulding • Mansel W. Griffiths • Steven P. Hooton • \\ Elizabeth M. Kutter · Andrew M. Kropinski · Ju-Hoon Lee • Martine Maes • Derek Pickard • \\ Sangryeol Ryu $\cdot$ Zargham Sepehrizadeh $\cdot$ S. Sabouri Shahrbabak • Ana L. Toribio • \\ Rob Lavigne
}

Received: 23 March 2012/ Accepted: 24 April 2012/Published online: 17 June 2012

(c) Springer-Verlag 2012

\begin{abstract}
We suggest a bacteriophage genus, "Viunalikevirus", as a new genus within the family Myoviridae. To date, this genus includes seven sequenced members: Salmonella phages ViI, SFP10 and ФSH19; Escherichia phages CBA120 and PhaxI; Shigella phage phiSboM-AG3; and Dickeya phage LIMEstone1. Their shared myovirus morphology, with comparable head sizes and tail dimensions, and genome organization are considered
\end{abstract}

Electronic supplementary material The online version of this article (doi:10.1007/s00705-012-1360-5) contains supplementary material, which is available to authorized users.

E. M. Adriaenssens · R. Lavigne $(\square)$

Laboratory of Gene Technology, Katholieke Universiteit

Leuven, Kasteelpark Arenberg 21-b2462,

3001 Heverlee, Belgium

e-mail: rob.lavigne@biw.kuleuven.be

E. M. Adriaenssens · M. Maes

Unit Plant-Crop Protection, Institute for Agricultural

and Fisheries Research, Burgemeester Van Gansberghelaan

96-b2, 9820 Merelbeke, Belgium

H.-W. Ackermann

Department of Microbiology, Immunology, and Infectiology, Faculty of Medicine, Laval University, Quebec,

QC G1X 4C6, Canada

H. Anany · M. W. Griffiths

University of Guelph, Canadian Research Institute for Food

Safety, Guelph, ON N1G 2W1, Canada

H. Anany

Microbiology Department, Faculty of Science,

Ain Shams University, Abbassia, Cairo, Egypt

B. Blasdel

Department of Microbiology,

The Ohio State University, Columbus,

OH 43210-1292, USA distinguishing features. They appear to have conserved regulatory sequences, a horizontally acquired tRNA set and the probable substitution of an alternate base for thymine in the DNA. A close examination of the tail spike region in the DNA revealed four distinct tail spike proteins, an arrangement which might lead to the umbrella-like structures of the tails visible on electron micrographs. These properties set the suggested genus apart from the recently ratified subfamily Tevenvirinae, although a significant evolutionary relationship can be observed.

\author{
I. F. Connerton · S. P. Hooton \\ Division of Food Sciences, University of Nottingham, \\ School of Biosciences, Sutton Bonington Campus, \\ Leicestershire LE12 5RD, UK \\ D. Goulding · D. Pickard · A. L. Toribio \\ The Wellcome Trust Sanger Institute, Genome Campus, \\ Hinxton, Cambridge CB10 1SA, UK \\ E. M. Kutter \\ The Evergreen State College, Olympia, WA 98505, USA
}

A. M. Kropinski

Laboratory for Foodborne Zoonoses, Public Health Agency

of Canada, 110 Stone Road West, Guelph, ON N1G 3W4,

Canada

A. M. Kropinski

Department of Molecular and Cellular Biology,

University of Guelph, Guelph, ON N1G 2W1, Canada

J.-H. Lee

Department of Food Science and Biotechnology,

Kyung Hee University, Yongin 446-701, South Korea 


\section{Introduction}

Bacteriophage classification is an evolving field that has traditionally been focused on morphology but has shifted more to genomics as DNA sequencing has become significantly less expensive. Accessible sequencing has resulted in a logarithmic increase in sequences deposited to databases and the need to classify them into new species, genera, subfamilies and families [1]. The subfamily category in the order Caudovirales is relatively new and was first introduced into the family Podoviridae with the creation of the subfamily Autographivirinae and the subfamily Picovirinae [2]. Similarly, within the family Myoviridae, the subfamily Tevenvirinae has been described, containing the genera T4likevirus and SchizoT4likevirus (http://www. ictvonline.org). Members of the first genus, with T4 as the type phage, are morphologically indistinguishable and comprise the former members of the T-even and pseudoT-even type phages [3]. The genus Schizot4likevirus, named after the historical schizo-T-even phage group, has as its type virus Vibrio phage KVP40 [4]. The phages belonging to this genus have larger head sizes than $\mathrm{T} 4$, an identical tail structure and over $20 \%$ of their coding sequences in common [4-6].

Many of the phages recognized as being T4-related by the marine ecology community, particularly a group of cyanomyoviruses, fall outside of these genera because their proteomes were considered to be too distinct in comparison to other clades within the viral domain. In a recent review of the "T4 supergroup of viruses," Petrov and coworkers [7] defined T4-related bacteriophages as possessing a Core Genome encoding approximately 37 proteins. Appropriately, we feel this definition not only includes the members of the genus T4likevirus and the genus SchizoT4likevirus but also various cyanomyoviruses and such phages as Campylobacter phages Cpt10 (FN667788) and CP220 (FN667789), Delftia phage $\Phi \mathrm{W}-14$ (NC_013697), and Salmonella phage ViI (NC_015296), each of which seems to be a prototype for a different genus within this supergroup.

S. Ryu

Department of Food and Animal Biotechnology,

Seoul National University, School of Agricultural

Biotechnology, Seoul 151-921, South Korea

Z. Sepehrizadeh $\cdot$ S. S. Shahrbabak

Department of Pharmaceutical Biotechnology,

Faculty of Pharmacy, Tehran University of Medical

Sciences, Tehran, Iran

Present Address:

A. L. Toribio

European Bioinformatics Institute,

Wellcome Trust Genome Campus Hinxton,

Hinxton, Cambridge CB10 1SD, UK
In this paper, we suggest the creation of a new genus "Viunalikevirus", after phage ViI, the first phage to be identified and studied of this viral genus [8]. This bacteriophage was first described in the 1930s as Typhoid phage Q151, which later became known as Vi1 [9-11] and Salmonella Typhi phage ViI [12], specific for the Vi antigen of Salmonella enterica subsp. enterica serovar Typhi. Genome analysis of six other phages, Salmonella phages SFP10 and ФSH19, Escherichia phages CBA120 and PhaxI, Shigella phage phiSboM-AG3 and Dickeya phage LIMEstone1, showed a DNA similarity of over $50 \%$ with each other and a distant relationship to T4 [13-17]. The close relatedness of these seven phages is examined in this paper, and the features of members of the newly suggested genus are described.

\section{Bacteriophages belonging to the genus "Viunalikevirus"}

The properties of the seven phages with fully sequenced and annotated genomes belonging to the genus "Viunalikevirus" are summarized in Table 1 . The distinguishing features of this genus include the genome size and organization, gene synteny, T4-like regulation of late transcription, the use of tail spikes for host recognition rather than T4-like tail fibers, and the use of a modified form of uracil (putatively HMdU) rather than thymine. The tail spikes of these phages were examined particularly closely, as this is the region in the genome that is most diverged. Morphologically near-identical phages occur in Acinetobacter, Bordetella, and Sinorhizobium, but their genomes have not yet been sequenced, and these phages are still awaiting classification.

\section{Morphology}

The phages of the genus "Viunalikevirus" have contractile tails and are thus members of the family Myoviridae. They are nearly indistinguishable in morphology, with similar head and tail dimensions, namely an isometric head of about $90 \mathrm{~nm}$ and a contractile tail of about $110 \times 18 \mathrm{~nm}$. The type phage ViI is depicted in Fig. 1. Based on this morphology, a number of other phages from the literature can be classified as probable viunalikeviruses (Table 1; Supplementary Figure 1). Heads are icosahedral, as indicated by the simultaneous presence of capsids with hexagonal and pentagonal outlines. Tails consist of a T4-like neck with a collar, a sheath surrounding a tail tube or core, a thin base plate, and an adsorption structure. The tail sheath has 24 transverse striations and resembles that of phage $\mathrm{T} 4$. The head and tail dimensions shown vary slightly, even when measured after magnification control by means of catalase crystals or $\mathrm{T} 4$ phage tails. The reasons are that micrographs were taken 
Table 1 Features of the phages belonging to the genus "Viunalikevirus"

\begin{tabular}{|c|c|c|c|c|c|c|c|c|c|c|}
\hline Phage name & Host & $\begin{array}{l}\text { Head } \\
\text { dimensions } \\
(\mathrm{nm})\end{array}$ & $\begin{array}{l}\text { Tail dimensions } \\
(\mathrm{nm})\end{array}$ & $\begin{array}{l}\text { Genome } \\
\text { size (bp) }\end{array}$ & $\mathrm{Mol} \% \mathrm{G}+\mathrm{C}$ & $\begin{array}{l}\text { Number } \\
\text { of ORFs } \\
\text { annotated }\end{array}$ & $\begin{array}{l}\text { Number } \\
\text { of } \\
\text { tRNAs }\end{array}$ & $\begin{array}{l}\text { Number } \\
\text { of } \\
\text { HEases }\end{array}$ & Reference & $\begin{array}{l}\text { Accession } \\
\text { number }\end{array}$ \\
\hline \multicolumn{11}{|c|}{ Phages with sequenced genomes } \\
\hline $\begin{array}{l}\text { ViI (syn. } \\
\text { Vi1, Vi01) }\end{array}$ & $\begin{array}{l}\text { Salmonella } \\
\text { Typhimurium }\end{array}$ & $90-95^{*}$ & $105-115 \times 17^{*}$ & 157,061 & 45 & 208 & 6 & & {$[8,11,12,29]$} & NC_015296 \\
\hline SFP10 & S. Typhimurium & $86^{*}$ & $109 \times 18$ & 157,950 & 44.5 & 201 & 4 & & [17] & HQ259103 \\
\hline ФSH19 & $S$. Typhimurium & 83 & $110 \times 14$ & 157,785 & 44.7 & 166 & 5 & 2 & [16] & JN126049 \\
\hline CBA120 & Escherichia coli & $90^{*}$ & $105 \times 17^{*}$ & 157,304 & 44.5 & 202 & 4 & & [15] & JN593240 \\
\hline PhaxI & E. coli & 80 & $115 \times 15$ & 156,628 & 44.5 & 209 & 4 & 1 & $\begin{array}{l}\text { Shahrbabak } \\
\text { et al, } \\
\text { unpublished }\end{array}$ & JN673056 \\
\hline LIMEstone 1 & $\begin{array}{c}\text { "Dickeya } \\
\text { solani" }\end{array}$ & $91^{*}$ & $114 \times 17^{*}$ & 152,427 & 44.9 & 201 & 1 & 14 & [13] & HE600015 \\
\hline $\begin{array}{l}\text { phiSboM- } \\
\text { AG3 }\end{array}$ & Shigella boydii & $83^{*}$ & $110 \times 14^{*}$ & 158,006 & 50.4 & 216 & 4 & & [14] & NC_013693 \\
\hline \multicolumn{11}{|c|}{ Phages with Vil morphology } \\
\hline 15,16 & S. Typhimurium & $88^{*}$ & $110-114 \times 17^{*}$ & - & - & - & - & & [27] & \\
\hline 10 & S. Typhimurium & $88^{*}$ & $112 \times 19^{*}$ & - & - & - & - & & [30] & \\
\hline FGCSSa1 & $S$. Typhimurium & 107 & $123 \times 17$ & - & - & - & - & & [31] & \\
\hline Det7 & $S$. Typhimurium & 90 & $120 \times 17$ & - & - & - & - & & [32] & \\
\hline 344 & $\begin{array}{l}\text { Serratia } \\
\quad \text { plymuthica }\end{array}$ & 85 & $117 \times 17$ & - & - & - & - & & $\begin{array}{l}\text { Grimont, } \mathrm{PhD} \\
\text { thesis }\end{array}$ & \\
\hline $\begin{array}{l}\text { E14, E1, E2, } \\
\text { E7 }\end{array}$ & $\begin{array}{l}\text { Acinetobacter } \\
\text { calcoaceticus }\end{array}$ & $91^{*}$ & $106 \times 17^{*}$ & - & - & - & - & & [33] & \\
\hline Ba5.2 & $\begin{array}{l}\text { Bordetella } \\
\text { avium }\end{array}$ & $80^{*}$ & $107 \times 17^{*}$ & - & - & - & - & & $\begin{array}{l}\text { Ackermann, } \\
\text { unpublished }\end{array}$ & \\
\hline $\begin{array}{l}\text { CM1 to } \\
\text { CM9, }\end{array}$ & $\begin{array}{l}\text { Sinorhizobium } \\
\text { meliloti }\end{array}$ & $98^{*}$ & $96-103 \times 16^{*}$ & - & - & - & - & & {$[34,35]$} & \\
\hline CM20, CM21 & & & & & & & & & & \\
\hline
\end{tabular}

* Measured after calibration with catalase crystals or T4 phage tails
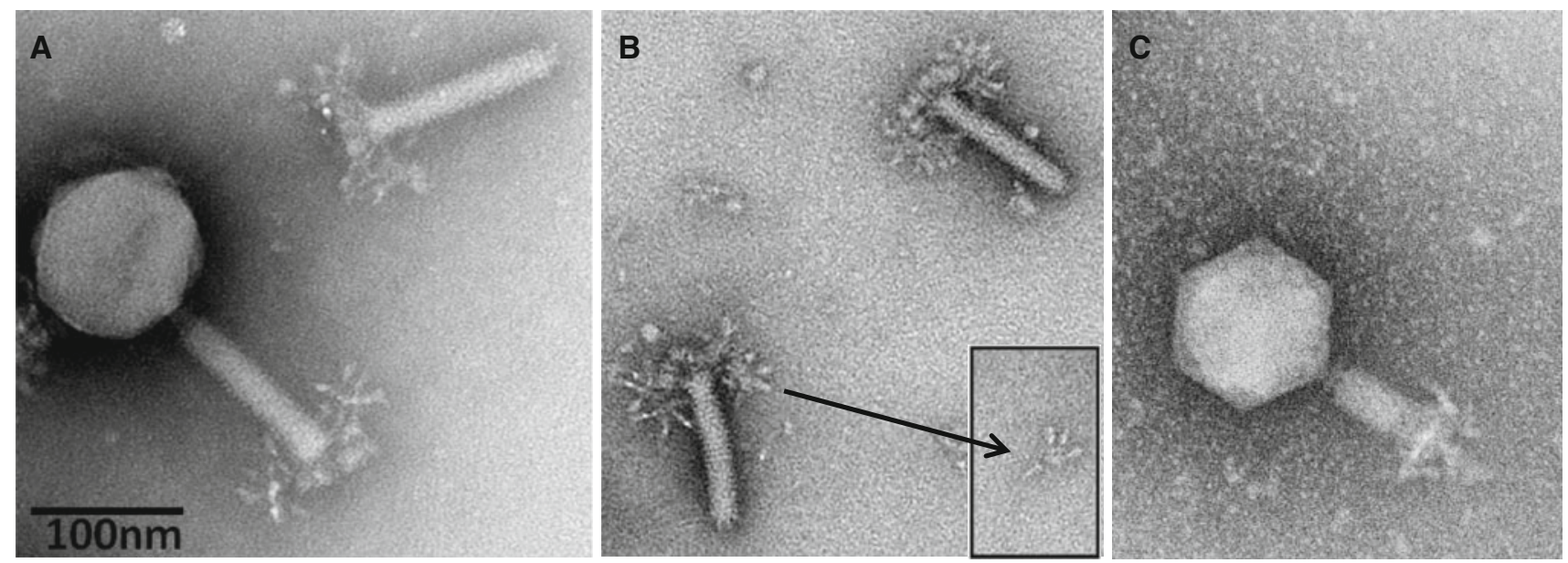

Fig. 1 Electron micrographs of Salmonella phage ViI. (A) Complete phage ViI with quiescent tail and severed tail; (B) ViI tails with unfolded tail entities, displaying an umbrella-like structure; (C) ViI with contracted tail. The phage was applied to a glow-discharged carbon/Formavar-coated 200-mesh copper grid and then stained with

over a period of over 40 years using different electron microscopes and that uranyl-acetate-stained capsids tend to be smaller than those of phosphotungstate-stained phages
$5 \%$ aqueous ammonium molybdate plus $1 \%$ trehalose. The grid was finally examined on a $120 \mathrm{KV}$ Philips Tecnai Spirit BioTwin transmission electron microscope fitted with a Tietz F415 chargecoupled-device (CCD) TemCam camera

(for references see Table 1). The collar is found in ViI-like phages of enterobacteria, Bordetella, and Sinorhizobium, but not of Acinetobacter. 
Fig. 2 Scale drawing of phage ViI with partially unfolded tail spikes. The sixth spike is behind the tail shaft and not shown. At present, the gene product responsible for the stalk, which attaches the star-like structures (and prongs) to the base-plate, has not been identified

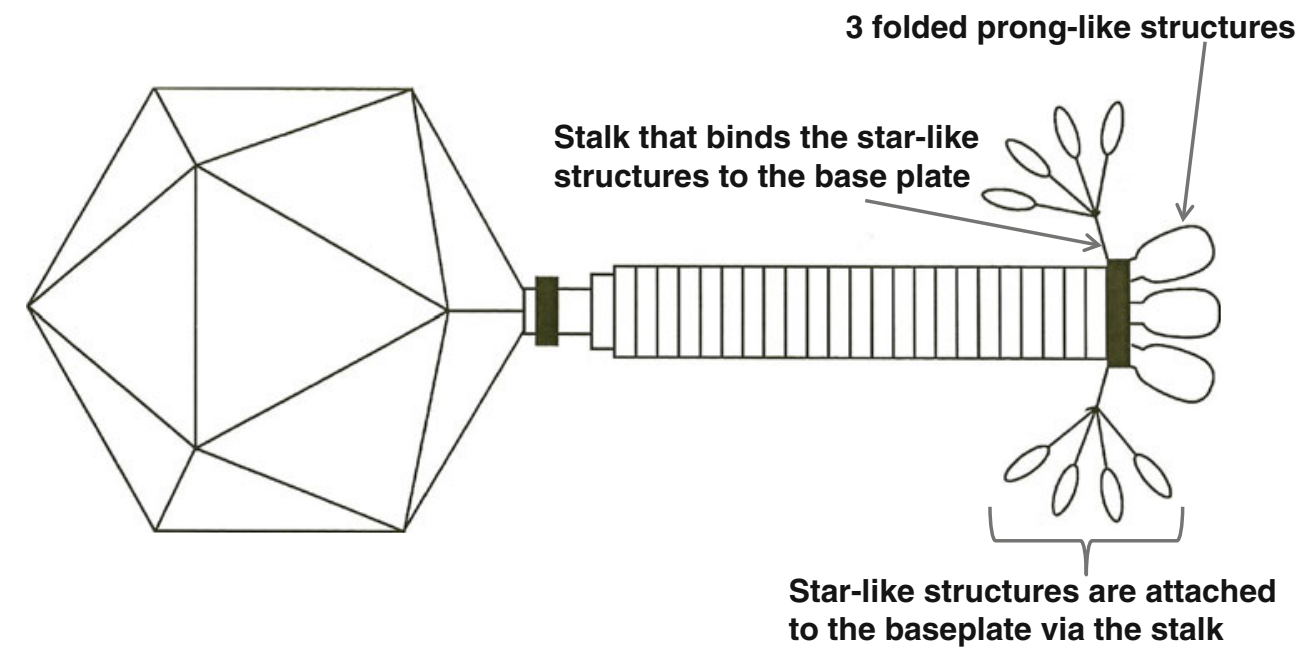

Table 2 Probable dimensions of the viunalikeviruses

\begin{tabular}{lll}
\hline Head/tail & Structure & Dimensions \\
\hline Head & Diameter & $90 \mathrm{~nm}$ between opposite apices \\
Tail & Overall & $108 \times 18 \mathrm{~nm}$ in the extended state \\
& Neck & $10 \times 8 \mathrm{~nm}$ \\
& Collar & $12 \times 3 \mathrm{~nm}$ \\
& Contracted sheath & $40 \times 25 \mathrm{~nm}$ \\
& Core & $8 \mathrm{~nm}$ wide, channel of $3 \mathrm{~nm}$ \\
& Base plate & $23 \times 3 \mathrm{~nm}$ \\
& Prongs & $17 \times 6 \mathrm{~nm}$ \\
& Stars & $30 \mathrm{~nm}$ wide \\
& Rays & $20 \mathrm{~nm}$ long \\
\hline
\end{tabular}

The adsorption organelle is complex and undergoes conformational changes. Quiescent tails show in profile 3-4 thick "prongs" with rounded ends (schematic representation in Fig. 2). In other instances, one observes a broad, umbrella-like, fibrous structure measuring 83-90 $\times 70-50 \mathrm{~nm}$ with star-like elements. The latter consists of 4 or 5 short filaments with bulbous tips (rays). Intermediate structures with both "prongs" and "stars" have been observed. Still other tails show, especially after tail contraction, an entangled mass of indistinct filaments. No similar tail structures have been reported in other phages. It is concluded that the tail has a six-folded symmetry and 6 "prongs" which unfold into as many "stars" and finally individual filaments. The most probable dimensions of the various ViI-like phages are indicated in Table 2.

\section{Comparative genomics}

Genome organization

The seven sequenced phages of the genus "Viunalikevirus" have genomes of similar size and share a high degree
Fig. 3 BLASTN comparison of the phages belonging to the suggested genus "Viunalikevirus". Phages are compared pairwise with their nearest neighbours in the figure. (A) Genomes are represented by a GC skew plot (top line for each phage), and annotated ORFs are depicted as boxes (bottom line for each phage). (B) Subregion pairwise comparison. ORFs are depicted as arrows according to frame

of sequence similarity. Sizes range from $152 \mathrm{~kb}$ for phage LIMEstone1 to $158 \mathrm{~kb}$ for phiSboM-AG3. Using the EMBOSS Stretcher algorithm (http://www.ebi.ac.uk/Tools/ psa/emboss_stretcher/), nucleotide similarities with the type phage ViI were computed. The Salmonella and Escherichia phages all shared $81 \%$ DNA identity; the similarity of ViI with phiSboM-AG3 and LIMEstone1 was $66 \%$ and $59 \%$, respectively. This high degree of similarity is clearly visible in Fig. 3, which shows a pair-wise BLASTN comparison between the different genomes, each phage compared with its nearest neighbour on the figure [18].

At the protein level, similarity was determined using CoreGenes [19] at its default settings. Relative to bacteriophage ViI, phage SFP10 has 189 proteins in common (91.0 \% similarity), PhaxI (90.4\%), CBA120 (88.5\%), phiSboM-AG3 (80.1\%), ФSH19 (76.9\%), and LIMEstone1 $(77.4 \%)$.

The gene order is strongly conserved in all seven phages, even though various functional regions are randomly distributed throughout the genome, in contrast to the fairly extensive functional clustering seen in tevenviruses [7]. This particular genome organization appears to be a distinguishing feature of this genus.

Regulatory elements

To investigate the existence of unique regulatory regions within the new genus, $100 \mathrm{bp}$ of 5 ' upstream sequence data 
A Phax $156,628 \mathrm{bp}$

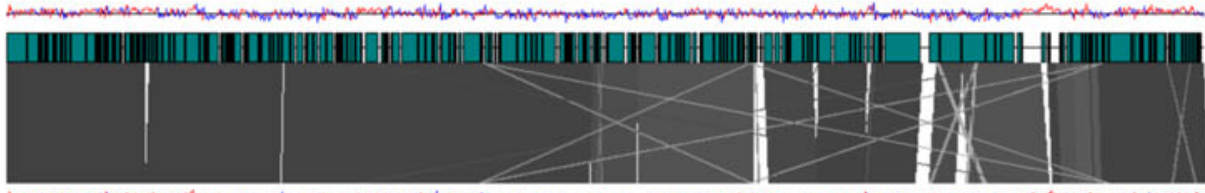

CBA120 157,304 bp

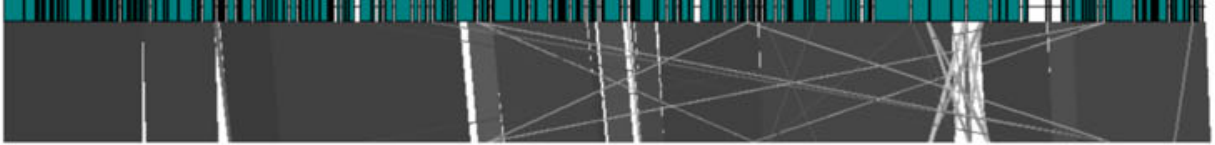

SFP10 A 157,950 bp

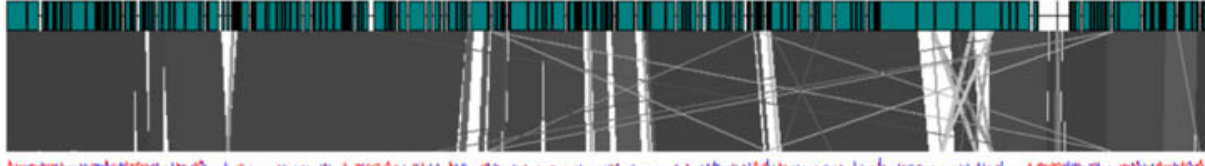

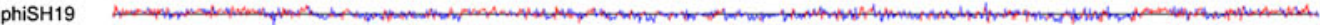
157.785 bs

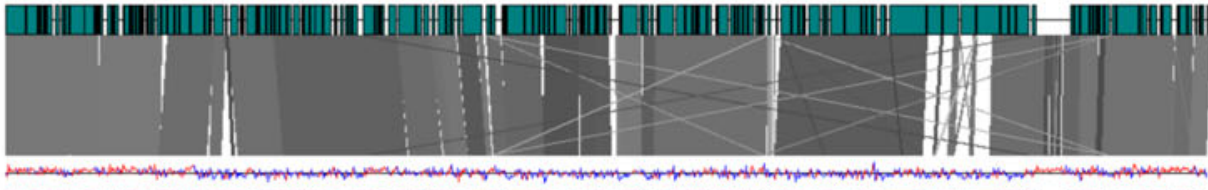

Vil

157.061 bp

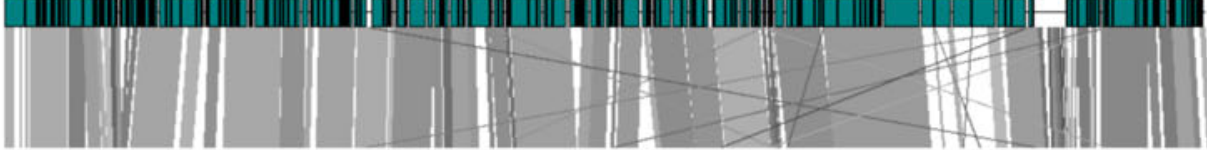

phiSboM-AG3 158,006 bp

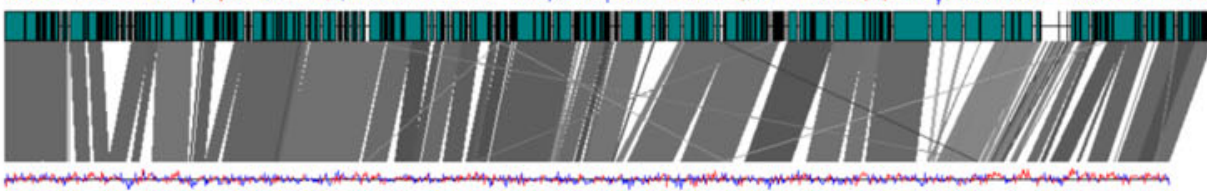

LIMEstone 1
152,427 bp
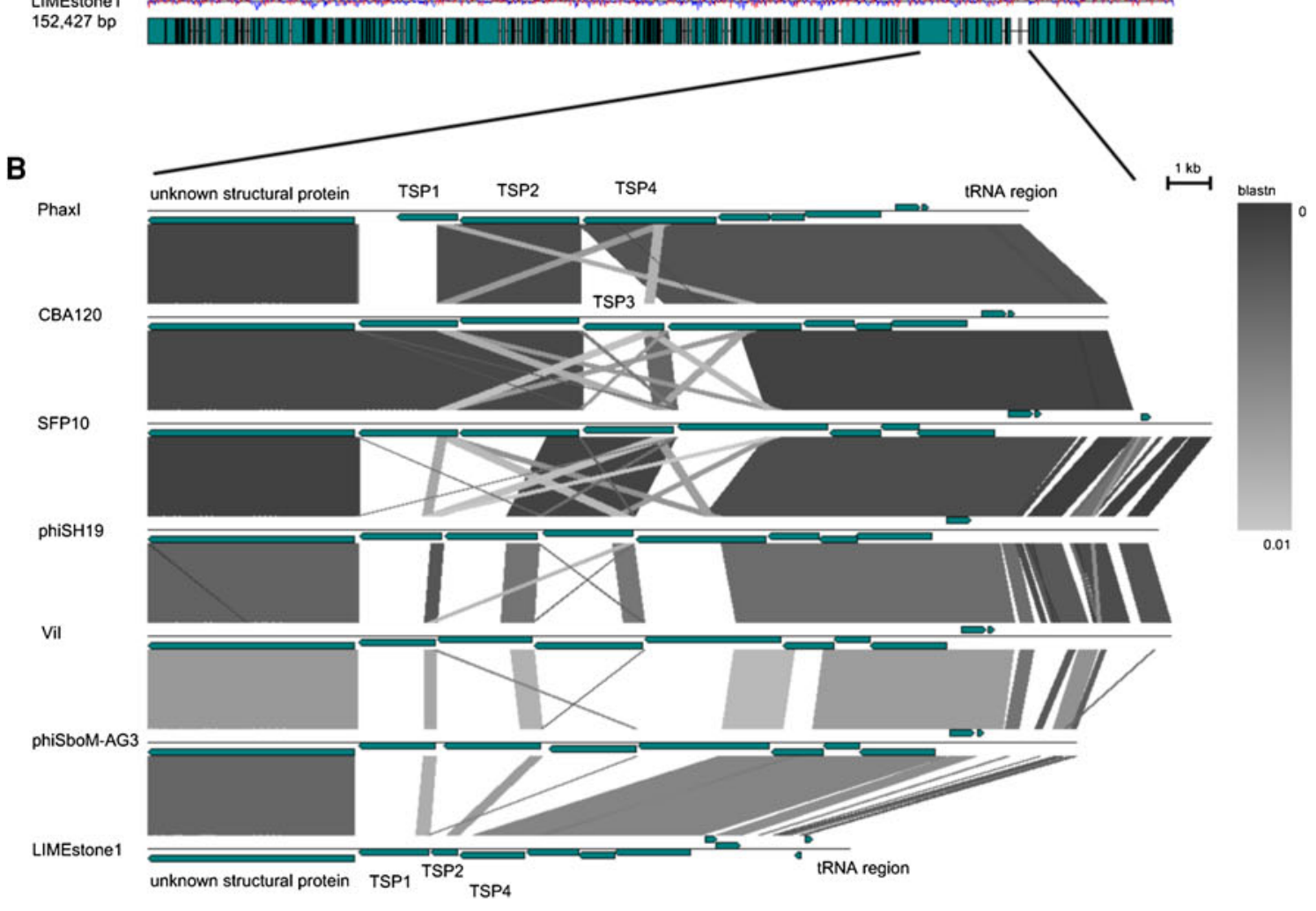
was extracted using extractUpStreamDNA (http://lfz. corefacility.ca/extractUpStreamDNA/ ) and submitted for MEME analysis [20] at http://meme.sdsc.edu/meme/cgibin/meme.cgi. Two motifs, with sequences TTCAAT [N14]TATAAT and CTAAATAcCcc, were found in all of the phages (Supplementary Figure 2). The core of the latter motif closely resembles the coliphage $\mathrm{T} 4$ late promoter core-TATAAATA [21, 22]; moreover, a number of these are located upstream of proven morphogenesis genes. Late transcription in T4 is dependent upon host polymerase and the products of genes 45 (RNA polymerase recruitment), 33 (co-activator of late transcription) and 55 (late promoter recognition protein). All of the phages in this genus possess homologs of these proteins; in the case of ViI, they are ViO1_135c, ViO1_069c, and ViO1_103c, respectively. In contrast, the sequence TTCAAT[N14]TATAAT is found upstream of many small conserved phage genes, reminiscent of the many immediate-early genes responsible for the transition from host to phage metabolism in phage T4 [23]. It is also found before various genes involved in DNA synthesis, including DNA polymerase, $n r d A$ and $B$, the gp45 sliding clamp and DNA ligase, suggesting that these sequences are also active in delayed early transcription [23]. The fact that they resemble but are not identical to the RpoD-dependent host promoters (TTGACA[N15-17]TATAAT) suggests that an unknown phage factor may be involved in their recognition.

\section{tRNAs and codon usage}

The ViI-related phages possess a collection of tRNA genes (Table 3) identified using tRNAscan-SE [24] and ARAGORN [25]. These tRNA genes are located at comparable positions within the ViI-like genomes (indicated in the bottom half of Fig. 3). For phages ViI, ФSH19, SFP10, CBA120, and PhaxI, the tRNA genes occupy regions that are associated with a perceptible increase over the average GC content (which peaks at around $60 \%$ ). A similar but less overt GC bias is observed in phiSboM-AG3, correlating with the higher mean GC content of this phage. In contrast, LIMEstone 1 possesses a solitary tRNA ${ }^{\text {Met }}$, found in a region that is slightly below the mean GC content. Based on the regional nucleotide sequence conservation and a common tendency to exhibit a localized increase in GC content, we hypothesize that a tRNA gene set comprising tRNA $^{\text {Met, }}$ tRNA $^{\text {Asn }}$, and tRNA ${ }^{\text {Ser }}$ was horizontally acquired by an ancestral ViI-like phage at some stage during its evolutionary history. The region also appears to be more amenable to the acquisition of further tRNA genes such as the tRNA ${ }^{\text {Gln }}$ in ViI, tRNA ${ }^{\text {Ile }}$ in CBA120, and tRNA ${ }^{\text {Ser }}$ (TCA) in SboM-AG3.

To examine the relationship between tRNA carriage and codon usage, relative synonymous codon usage and codon adaptation indices were generated for each phage using E-CAI [26]. Examination of the codon usage of the ViI-like phages does not reveal any apparent link between codon preference and the presence of their cognate tRNAs. A wider examination of the ViI-like phages and host bacteria codon usage tables indicated that the codons specified by the ViI-like phage tRNAs are actually amongst the most frequently occurring in the host genomes. These data suggest that the requirements of the phage could be served by sufficient host tRNAs.

\section{DNA modification}

None of the seven sequenced viunalikeviruses carry any of the genes responsible for substituting $\mathrm{C}$ for hydroxymethylcytosine (HMdC) in the DNA of T4 as well as the more closely related T4-like phages, implying that these phages all use $\mathrm{C}$ in their DNA. However, while most of the genes involved in nucleotide metabolism appear homologous to corresponding genes in tevenviruses, the ORF originally annotated as a thymidylate synthase in most viunalikeviruses is much more closely related to the hydroxymethyluracil (HMdU) transferases found in a few other phages, such as Delftia phage $\Phi \mathrm{W}-14$ and Bacillus subtilis phage

Table 3 tRNAs and their codons of the phages of the genus "Viunalikevirus"

\begin{tabular}{|c|c|c|c|c|c|c|c|c|}
\hline \multirow[b]{2}{*}{ Phage } & \multicolumn{8}{|c|}{ tRNA $^{\mathrm{a}}$ (Codon) } \\
\hline & Met (ATG) & Ser 1 (TCA) & Asn (AAC) & Ile (ATC) & Gln (CAA) & Tyr (TAC) & Ser 2 (AGC) & Undetermined (???) \\
\hline ViI & $+(+)$ & $-(-)$ & $+(+)$ & $-(-)$ & $+(+)$ & $+(+)$ & $+(+)$ & $+(-)$ \\
\hline phiSboM-AG3 & $-(-)$ & $+(+)$ & $+(+)$ & $-(-)$ & $-(-)$ & $+(+)$ & $+(+)$ & $-(-)$ \\
\hline CBA120 & $+(+)$ & $-(-)$ & $+(+)$ & $-(+)$ & $-(-)$ & $-(-)$ & $+(+)$ & $+(-)$ \\
\hline ФSH19 & $+(+)$ & $-(-)$ & $+(+)$ & $-(-)$ & $-(-)$ & $+(+)$ & $+(+)$ & $+(-)$ \\
\hline SFP10 & $+(+)$ & $-(-)$ & $+(+)$ & $-(-)$ & $-(-)$ & $-(-)$ & $+(+)$ & $+(-)$ \\
\hline LIMEstone1 & $+(+)$ & $-(-)$ & $-(-)$ & $-(-)$ & $-(-)$ & $-(-)$ & $-(-)$ & $-(-)$ \\
\hline PhaxI & $+(+)$ & $-(-)$ & $+(+)$ & $-(-)$ & $-(-)$ & $-(-)$ & $+(+)$ & $+(-)$ \\
\hline
\end{tabular}

a + present; - absent, non-bracketed-identified with tRNAscan-SE, brackets-identified with ARAGORN 
Fig. 4 Dendogram of hydroxymethyluridylate transferase sequences of members of the genus "Viunalikevirus" compared with those of known hydroxymethyluridylate transferases as well as thymidylate synthase sequences from other phages. Proteins were aligned in MAFFT, with BLOSUM 30 used as the scoring matrix. The topology of the tree is based on $\mathrm{NJ}$ bootstrap values, shown above, which were calculated using the Poisson distance between sequences over 100 pseudoreplicates

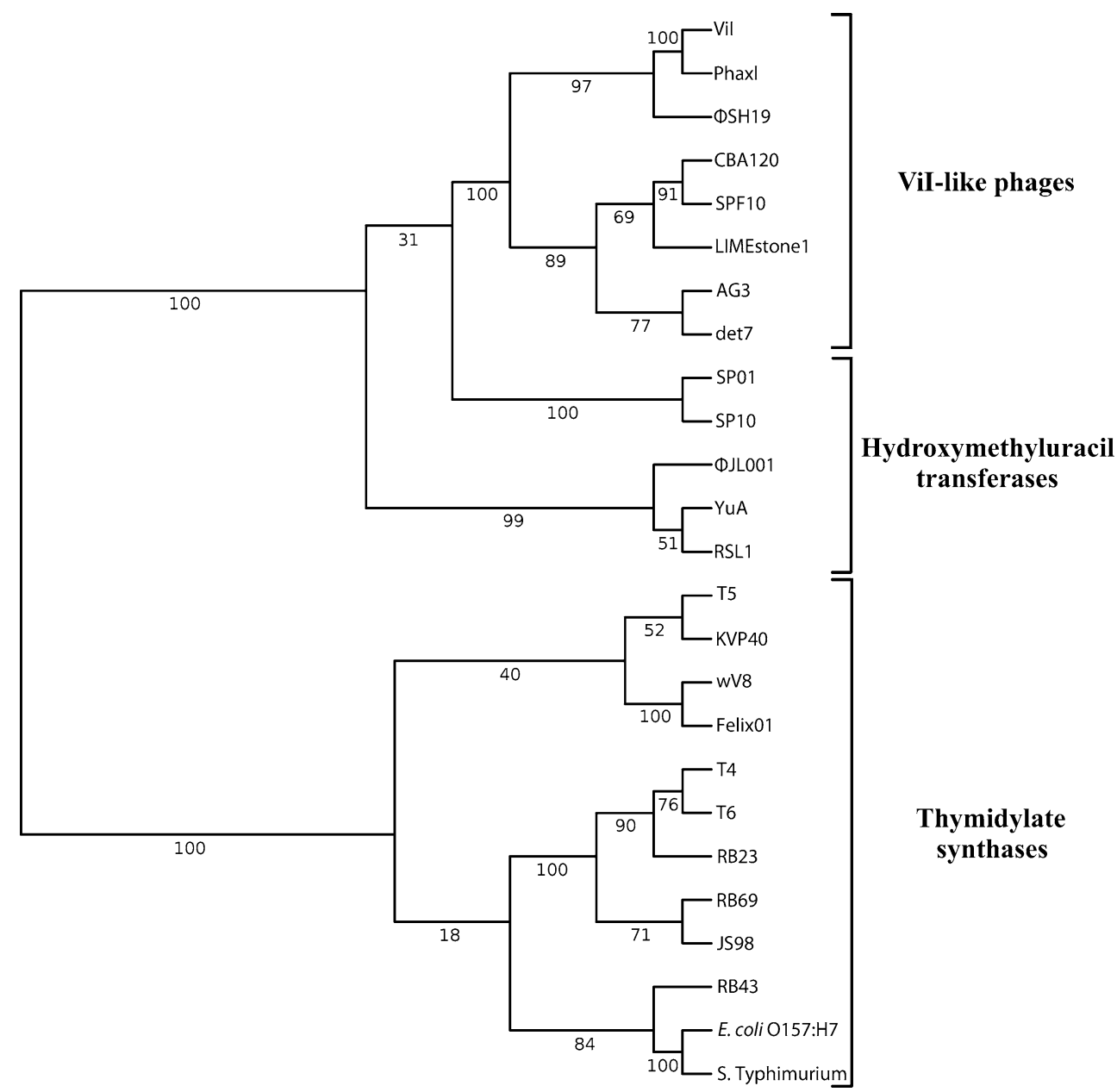

Tail spikes

SP01 (Fig. 4). This appears to be a primary distinguishing feature of the new genus "Viunalikevirus".

The incorporation of a non-canonical nucleotide in place of thymine was first suspected when Kutter et al. [15] found that CBA120 could not incorporate tritiated thymidine into its DNA, while still making hundreds of phage per cell. To investigate the nature of this probable substitution, an assay was constructed that took advantage of the characterized methylation sensitivities of five commercially available restriction endonucleases according to the manufacturer's instructions. Testing showed that both CBA120 and LIMEstone1 genomic DNA are sensitive to cleavage by restriction endonucleases that have been previously shown to be active against fully substituted HMdUcontaining DNA but not endonucleases that are inhibited by that modification. Using this assay, we were able to distinguish HMdU-containing DNA from DNA containing canonical nucleotides as well as other non-canonical substitutions such as methylcytosine, hydroxymethylcytosine, glycosylated hydroxymethylcytosine, uracil, and phosphoglucuronated 5-(4',5'-dihydroxypentyl) uracil (Blasdel, Brabban and Kutter, unpublished observations).
Bacteriophages within the genus "Viunalikevirus" are closely related to each other in terms of their DNA sequence and the remarkable synteny of the vast majority of genes along their entire genomes (see Fig. 3). The sole region of significant divergence lies within the tail spike region of each phage (bottom half of Fig. 3), yet even within these regions, there are significant zones of homology at both the DNA and protein level. This arrangement of tail spike genes within their genomes is a unique signature of this group of phages.

As more representatives of this phage genus have been identified, variants in the number of tail spike proteins (TSPs) have been found. For example, LIMEstone1 and PhaxI have one and three likely tail spike proteins, respectively. Most of the identified ViI-like phages to date are now believed to encode four tail spike proteins, as illustrated in Fig. 3. Many of the different modules and domains we have identified in TSP1-4 have been discussed in detail previously $[8,15,16]$. Briefly, tail spike proteins 1 to 3 (TSPs1-3) have a number of salient shared features and modules, while TSP4 
Fig. 5 A generalised depiction of the various domains and regions identified within the tail spike proteins (TSPs) of members of the genus

"Viunalikevirus"

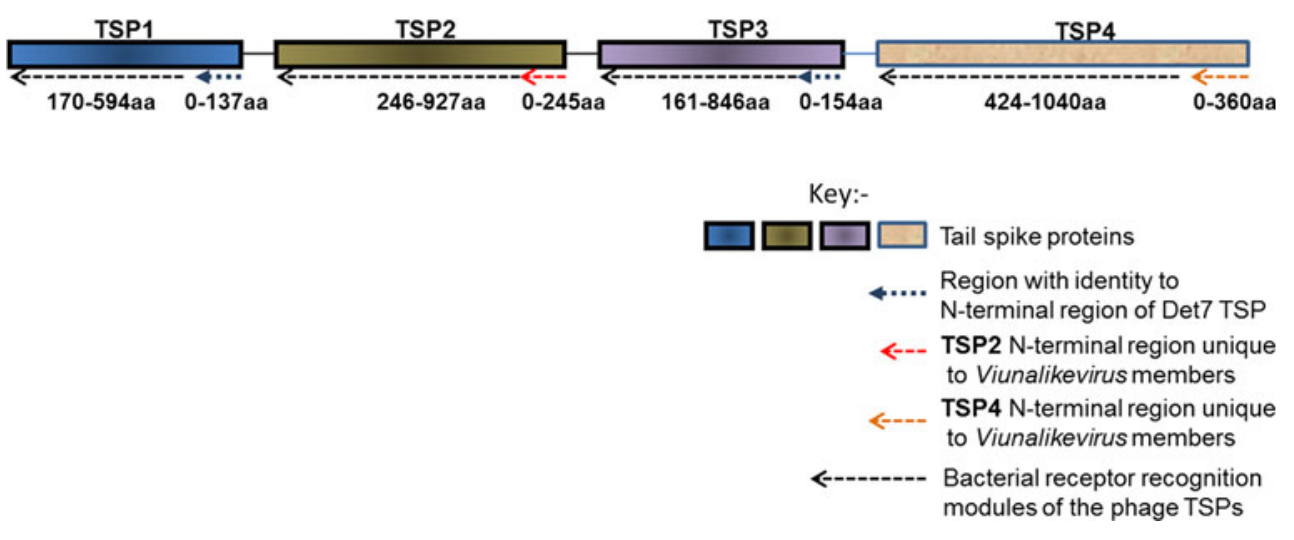

The sequence data clearly point to up to four distinct TSPs, and the EM morphology, as shown in Fig. 1B with its small inserted image, appears to support this. There are four spikes surrounding a less dense central core, reminiscent of tail structures associated with podoviruses when seen separated from the phage head [8]. This central core could well be the tail stalk that binds the spikes to the base plate itself. The overall phage morphology is summarized in Fig. 2, but aspects of the tail spike structure require further investigation for each phage.

Table 4 summarizes the known and putative targets of the TSPs. The range typically encompasses both plant and animal pathogens of the family Enterobacteriaceae. Many of the TSP targets have been identified experimentally, but a number were putatively identified using BLASTP when the criteria included both very good $\mathrm{E}$ values and coverage of at least $70 \%$ of the length of the TSP. In most cases, the putative homologies to the ViI-like TSPs were to tail spike genes from temperate phages. For example, the unique TSP1 of phiSboM-AG3 is very similar to a gene found in an uncharacterized temperate phage of $E$. coli UMNK88 with the accession number AEE57668 (266/428aa identity; 325/428aa positives). A further interesting example is TSP4 from phages CBA120 and PhaXI that is similar to a further uncharacterized lysogenic phage gene (accession number CBJ02120), this time located in E.coli H10407 (247/375aa identity; 299/375aa positives). It may not be a coincidence that E. coli $\mathrm{H} 10407$ is an $\mathrm{O} 78$ antigenic type strain, as this was also the serotype of the only member of the ECOR collection that CBA120 infected [15].

Given the remarkable synteny observed in these phages, other genes may be conserved that have a pan-global inhibitory effect on their enteric hosts. These will be a target in future research.

\section{Phylogeny}

To further explore this new group of ViI-like phages and to differentiate them from other phages, phylogenetic analysis 


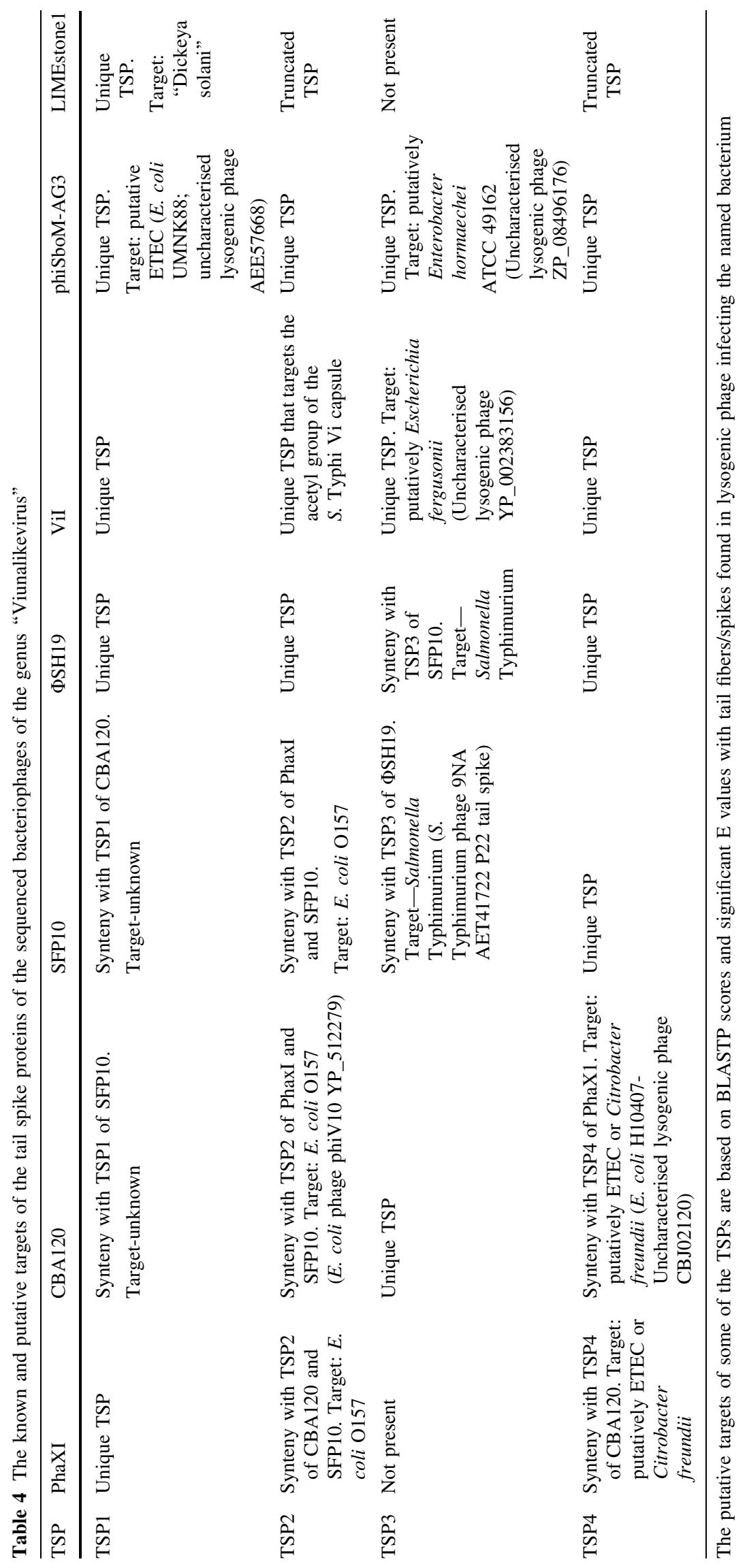




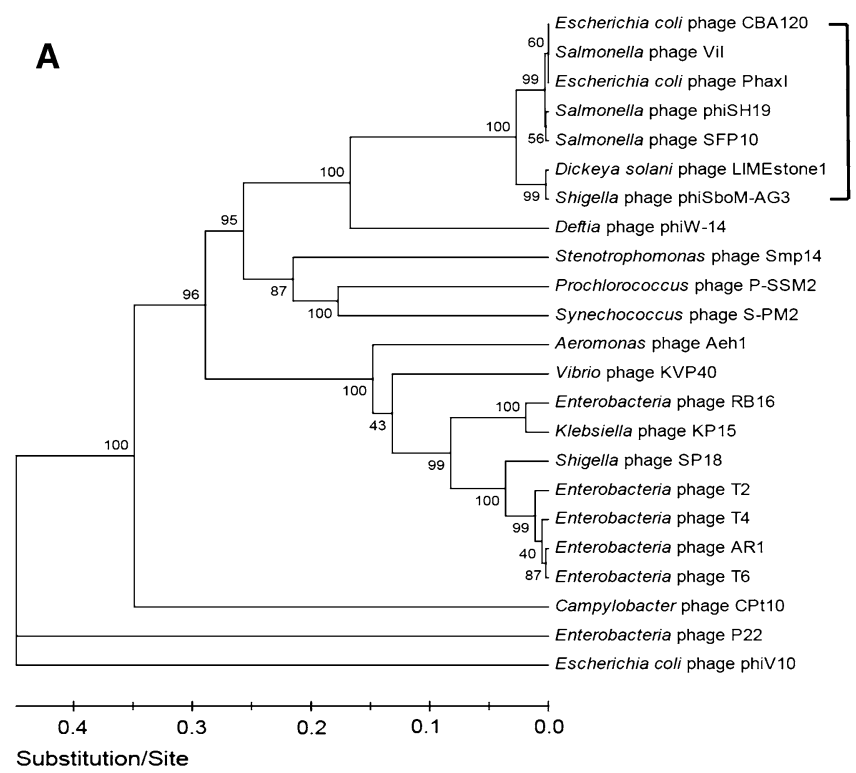

C
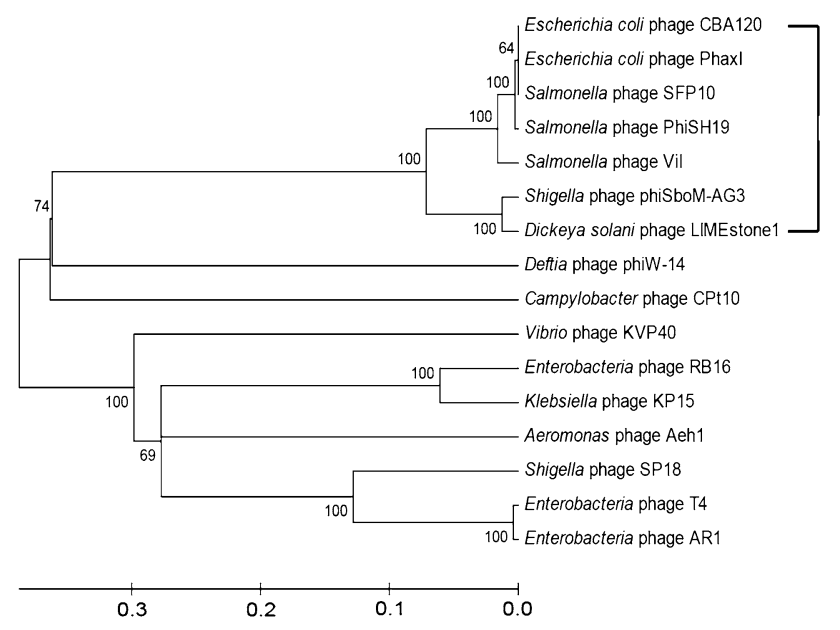

Substitution/Site

Fig. 6 Phylogenetic trees. Comparisons of (A) major capsid proteins (T4 gp23 homologues), (B) DNA polymerases (T4 gp43 homologues), (C) DNA ligases (T4 gp30 homologues), and (D) terminase

of highly conserved proteins such as the major capsid proteins (T4 gp23 homologues), DNA polymerases (T4 gp43 homologues), DNA ligases (T4 gp30 homologues), and terminase large subunits (T4 gp17 homologues) was carried out (Fig. 6). All of these analyses substantiated the establishment of the new phage genus "Viunalikevirus" and established the same pattern of their relationship to the tevenviruses and other T4-related phages (S-PM2, P-SSM2). Interestingly, while Delftia acidovorans phage $\Phi \mathrm{W}-14$ is not a member of this new phage group, it is most
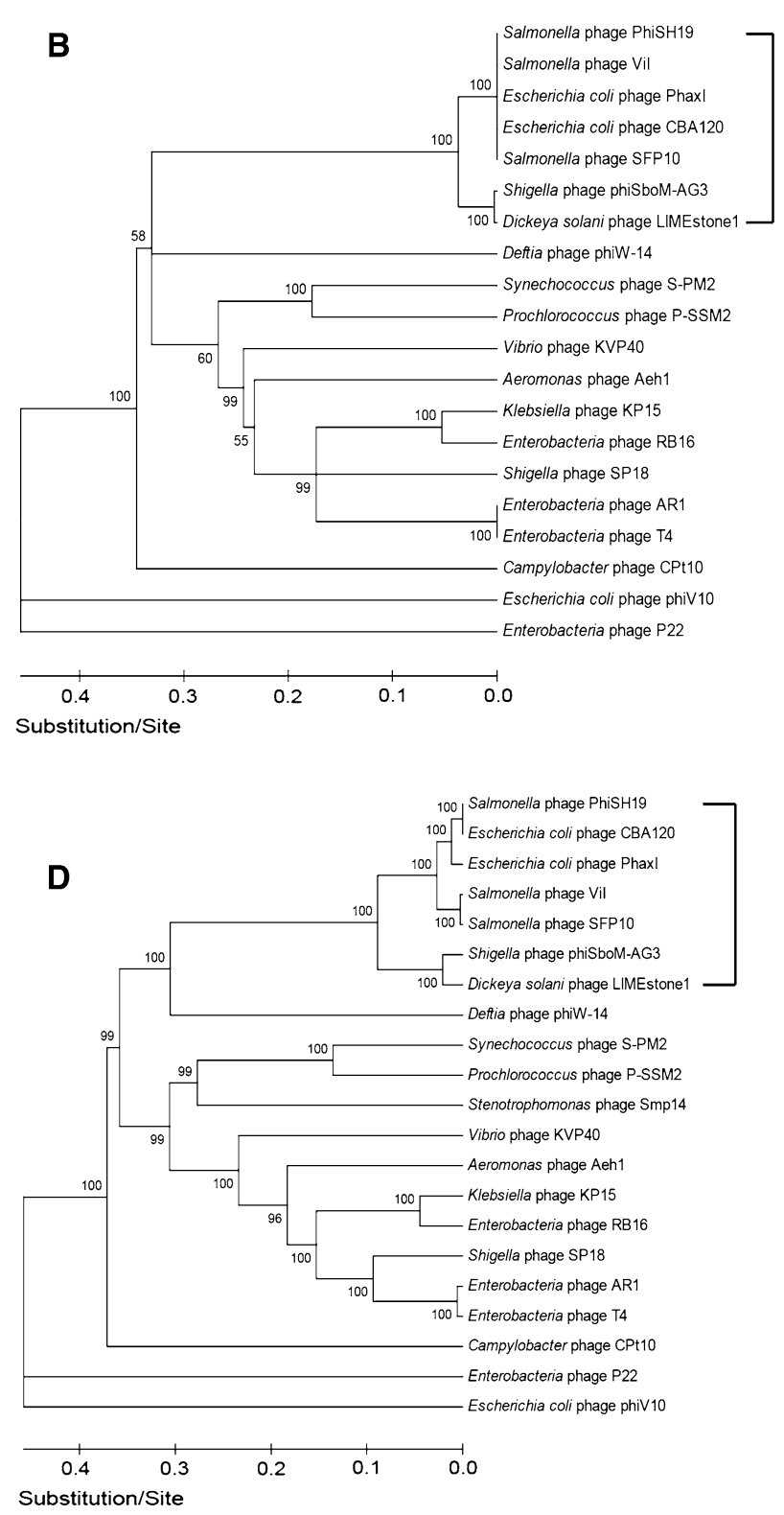

large subunits (T4 gp17 homologues). Square brackets indicate the suggested genus "Viunalikevirus"

closely related to the ViI-like phages in all of these phylogenetic analyses. Also of interest, this has led to the somewhat surprising finding that Shigella phage phiSboMAG3 is much more closely related to Dickeya phage LIMEstone1 than it is to the other enteric phages.

These findings could ultimately lead to a reclassification of the higher orders of bacterial viruses, but at present, the genus "Viunalikevirus" will be an independent genus in the family Myoviridae, despite the relationship of its members to the phages of the subfamily Tevenvirinae. 


\section{Discussion}

The group of ViI-like phages differs from any established phage genus by its characteristic morphology and genomic properties. It stands virtually alone in the phage world in a number of ways despite its distant relationships to T4-like phages. The phages share a large degree of DNA homology, a similar genome organization, the presence of a modified base (suspected to be HMdU), and conserved patterns of early and late promoter sequences. Furthermore, there seems to be a horizontally acquired tRNA set conserved among most ViI-like phages. We suggest the term "Viunalikevirus" as the taxonomic name for this new genus.

Combining the morphology information of the EMs and the sequence data of the tail spike regions of these phages, new hypotheses can be made about the morphology of the ViI-like tail structure. Figure 1A shows the general morphology of the ViI-like bacteriophages, with six apparent tail spike entities. Some of these can be seen to be unfolded, while others are not, creating "stars" and "prongs", respectively. Figure $1 \mathrm{~b}$ shows the arrangement of the tail spikes in more detail with respect to most of the ViI-like family, where we now hypothesize that up to four spikes are present on each of the six tail entities, encoded by four tail spike genes (TSP1-4) (as represented schematically in Fig. 2). In this respect, comparisons to the EM of LIMEstone1 are illuminating (Supplementary Figure 3), since it encodes just one complete tail spike protein (TSP1) and a truncated TSP4. The overall structure is less elaborate, and the tail spikes are less complex, showing no splitting into the umbrella-like arrangement characteristic of ViI and several of the others, as seen in Fig. 1A and B, for example.

Figure 3 provides extensive evidence that regions of DNA are shared by the various TSPs, both within the same phage and among the other viunalikeviruses. These homologous regions are most likely involved in the domain exchange evident in this phage family within the tail spike repertoire. Such re-assortment of tail spike receptor recognition 'modules' would be further enhanced by having so many TSPs and shared enteric hosts. For example, a substantial region of DNA (278 bases over a range of 386 bases-72\% match) from TSP1 of PhaXI is also located in TSP4, and regions like these can serve as recombination hot spots for domain exchange.

A result of the broad tail spike repertoire and putative targets (Table 4) might be that the host range of some of the ViI-like phages is more extensive than previously considered. This was already witnessed for SFP10, which infects both Salmonella Typhimurium and E. coli O157. For phages PhaXI and CBA120, a shared TSP2 was found with ETEC or Citrobacter freundii as potential targets, and TSP1 of CBA120 and SFP10 showed synteny, with the target of the tail spike unknown. So far, for all the sequenced ViI-like phages, unique TSPs are present for which the targets have not yet been identified. At the same time, their specificity can be highly targeted. For example, CBA120 does not target any of the common laboratory E. coli strains, and only hits one member of the ECOR collection (ECOR70) in addition to hitting most tested E. coli O157 strains [15]. It also does not target most Salmonella strains; only one (very weak) hit has been found to date, in subspecies indica [15]. Determining the roots of this host specificity will be an interesting area for future research.

If these ViI-like phages have a capacity to infect such diverse enteric hosts, they will need to have mechanisms for overcoming an equally diverse range of bacterial restriction and modification systems. The probable replacement of thymidine (dThd) with HMdU appears to be one such method employed by this phage genus to circumvent the host's restriction and modification pathways. Aside from defense against some restriction enzymes, it is not clear what, if any, evolutionary advantages the incorporation of HMdU provides for viunalikeviruses. However, for Bacillus phage SPO1, the middle-mode genes are preferentially transcribed from DNA that has HMdU rather than dThd [28]. Many members of the related genus T4likevirus also use the presence of HMdC in their DNA to rapidly block all transcription of cytosine-containing DNA and to much more gradually degrade all cytosine-containing DNA without damaging their own DNA [23].

We conclude that the creation of the genus "Viunalikevirus" is another appropriate step towards phage classification.

Acknowledgments AMK would like to thank Andre Villegas for developing "extractUpStreamDNA". BB and EMK would like to thank NIH grant 2R15GM63637-3A1 for support, along with the many members of the Evergreen Phage lab who participated in these studies. DG, DP and ALT are very grateful for funding from the Wellcome Trust. RL and AMK are respectively the chair and vicechair of the ICTV Prokaryotic Virus Subcommittee.

Conflict of interest The authors declare that they have no conflict of interest.

\section{References}

1. King AMQ, Lefkowitz E, Adams MJ, Carstens EB (2012) Virus taxonomy: ninth report of the international committee on taxonomy of viruses. Academic Press, London

2. Lavigne R, Seto D, Mahadevan P, Ackermann H-W, Kropinski AM (2008) Unifying classical and molecular taxonomic classification: analysis of the Podoviridae using BLASTP-based tools. Res Microbiol 159: 406-414. doi:10.1016/j.resmic.2008.03.005 
3. Desplats C, Krisch HM (2003) The diversity and evolution of the T4-type bacteriophages. Res Microbiol 154: 259-267. doi: 10.1016/S0923-2508(03)00069-X

4. Miller ES, Heidelberg JF, Eisen JA, Nelson WC, Durkin AS, Ciecko A, Feldblyum TV, White O, Paulsen IT, Nierman WC, Lee J, Szczypinski B, Fraser CM (2003) Complete genome sequence of the broad-host-range vibriophage KVP40: comparative genomics of a T4-related bacteriophage. J Bacteriol 185:5220-5233

5. Ackermann H-W, Krisch HM (1997) A catalogue of T4-type bacteriophages. Arch Virol 142:2329-2345

6. Matsuzaki S, Tanaka S, Koga T, Kawata T (1992) A broad-hostrange vibriophage, KVP40, isolated from sea water. Microbiol Immunol 36:93-97

7. Petrov VM, Ratnayaka S, Nolan JM, Miller ES, Karam JD (2010) Genomes of the T4-related bacteriophages as windows on microbial genome evolution. Virol J 7. doi:10.1186/1743422X-7-292

8. Pickard D, Toribio AL, Petty NK, van TA, Yu L, Goulding D, Barrell B, Rance R, Harris D, Wetter M, Wain J, Choudhary J, Thomson N, Dougan G (2010) A conserved acetyl esterase domain targets diverse bacteriophages to the Vi capsular receptor of Salmonella enterica serovar Typhi. J Bacteriol 192: 5746-5754. doi:10.1128/JB.00659-10

9. Craigie J, Brandon KF (1936) Bacteriophage specific for the O-resistant V form of B. typhosus. J Pathol 43:233-248. doi: 10.1002/path.1700430202

10. Craigie J, Yen CH (1937) V Bacteriophages for B. typhosus. Trans Royal Soc Can 5:79-87

11. Bradley DE, Kay D (1960) The fine structure of bacteriophages. J General Microbiol 23:553-563

12. Ackermann H-W, Berthiaume L, Kasatiya SS (1970) Ultrastructure of Vi phages I to VII of Salmonella typhi. Can J Microbiol 16:411-413

13. Adriaenssens EM, Van Vaerenbergh J, Vandenheuvel D, Dunon V, Ceyssens PJ, De Proft M, Kropinksi AM, Noben JP, Maes M, Lavigne R (2012) T4-related bacteriophage LIMEstone isolates for the control of soft rot on potato caused by 'Dickeya solani'. PLoS ONE 7:e33227. doi:10.1371/journal.pone.0033227

14. Anany H, Lingohr E, Villegas A, Ackermann HW, She YM, Griffiths M, Kropinski A (2011) A Shigella boydii bacteriophage which resembles Salmonella phage ViI. Virol J 8:242. doi: 10.1186/1743-422X-8-242

15. Kutter EM, Skutt-Kakaria K, Blasdel B, El-Shibiny A, Castano A, Bryan D, Kropinski AM, Villegas A, Ackermann HW, Toribio AL, Pickard D, Anany H, Callaway T, Brabban AD (2011) Characterization of a ViI-like phage specific to Escherichia coli O157:H7. Virol J 8:430. doi:10.1186/1743-422X-8-430

16. Hooton SP, Timms AR, Rowsell J, Wilson R, Connerton IF (2011) Salmonella Typhimurium-specific bacteriophage PhiSH19 and the origins of species specificity in the Vi01-like phage family. Virol J 8:498. doi:10.1186/1743-422X-8-498

17. Park M, Lee JH, Shin H, Kim M, Choi J, Kang DH, Heu S, Ryu S (2011) Characterization and comparative genomic analysis of a novel bacteriophage SFP10 simultaneously inhibiting both Salmonella and Escherichia coli O157:H7. Appl Environ Microbiol. doi:10.1128/AEM.06231-11
18. Sullivan MJ, Petty NK, Beatson SA (2011) Easyfig: a genome comparison visualizer. Bioinformatics 27. doi:10.1093/ bioinformatics/btr039

19. Zafar N, Mazumder R, Seto D (2002) CoreGenes: a computational tool for identifying and cataloging "core" genes in a set of small genomes. BMC Bioinform 3:12

20. Bailey TL, Elkan C (1994) Fitting a mixture model by expectation maximization to discover motifs in biopolymers. AAAI Press, Menlo Park, pp 28-36

21. Nechaev S, Geiduschek EP (2008) Dissection of the bacteriophage T4 late promoter complex. J Mol Biol 379:402-413

22. Geiduschek EP, Kassavetis GA (2010) Transcription of the T4 late genes. Virol J 7:288

23. Miller ES, Kutter E, Mosig G, Arisaka F, Kunisawa T, Ruger W (2003) Bacteriophage T4 genome. Microbiol Mol Biol Rev 67:86-156

24. Schattner P, Brooks AN, Lowe TM (2005) The tRNAscan-SE, snoscan and snoGPS web servers for the detection of tRNAs and snoRNAs. Nucleic Acids Res 33: W686-W689. doi:10.1093/nar/ gki366

25. Laslett D, Canback B (2004) ARAGORN, a program to detect tRNA genes and tmRNA genes in nucleotide sequences. Nucleic Acids Res 32:11-16. doi:10.1093/nar/gkh152

26. Puigbo P, Bravo IG, Garcia-Vallve S (2008) E-CAI: a novel server to estimate an expected value of Codon Adaptation Index (eCAI). BMC Bioinform 9:65. doi:10.1186/1471-2105-9-65

27. Ackermann H-W, Gershman M (1992) Morphology of phages of a general Salmonella typing set. Res Virol 143:303-310

28. Stewart CR, Casjens SR, Cresawn SG, Houtz JM, Smith AL, Ford ME, Peebles CL, Hatfull GF, Hendrix RW, Huang WM, Pedulla ML (2009) The genome of Bacillus subtilis bacteriophage SPO1. J Mol Biol 388:48-70. doi:10.1016/j.jmb.2009. 03.009

29. Ackermann H-W, DuBow MS (1987) Viruses of prokaryotes. CRC Press, Boca Raton

30. Demczuk W, Ahmed R, Ackermann H-W (2004) Morphology of Salmonella enterica serovar Heidelberg typing phages. Can J Microbiol 50:873-875. doi:10.1139/w04-075

31. Carey-Smith GV, Billington C, Cornelius AJ, Hudson JA, Heinemann JA (2006) Isolation and characterization of bacteriophages infecting Salmonella spp. FEMS Microbiol Lett 258: 182-186. doi:10.1111/j.1574-6968.2006.00217.x

32. Walter M, Fiedler C, Grassl R, Biebl M, Rachel R, Hermo-Parrado XL, Llamas-Saiz AL, Seckler R, Miller S, van Raaij MJ (2008) Structure of the receptor-binding protein of bacteriophage det7: a podoviral tail spike in a myovirus. J Virol 82:2265-2273. doi:10.1128/JVI.01641-07

33. Ackermann H-W, Brochu G, Emadi Konjin HP (1994) Classification of Acinetobacter phages. Arch Virol 135:345-354

34. Krsmanovic-Simic D, Werquin M (1973) Study of bacteriophages of Rhizobium meliloti. C R Acad Sci Hebd Seances Acad Sci D 276:2745-2748

35. Werquin M, Ackermann H-W, Levesque RC (1988) A Study of 33 Bacteriophages of Rhizobium meliloti. Appl Environ Microbiol 54:188-196 